\title{
PÉRDIDA DE HUMEDAD Y ABSORCIÓN DE ACEITE EN LA FRITURA A VACÍO DE UN PRODUCTO CÁRNICO TIPO CHORIZO CON INCORPORACIÓN DE HARINA DE GARBANZO (CICER ARIETUM)
}

\section{LOSS OF MOISTURE AND OIL ABSORPTION ON FRYING ON A VACUUM OF A MEAT PRODUCT TYPE SAUSAGE WITH CHICKPEA FLOUR INCORPORATION (CICER ARIETUM)}

\section{Acevedo Correa*, D; Montero Castillo, P; Torres Díaz L; Torres Díaz S.}

Universidad de Cartagena/Programa de Ingeniería de Alimentos/Grupo de investigación e innovación y desarrollo agropecuario y agroindustrial (IDAA)/Cartagena Bolívar/Colombia. *Correo electronico:diofanor3000@gmail.com

Recibido mayo 21 de 2017 ; Aprobado 11 de octubre de 2017

\section{RESUMEN}

Dentro de los tratamientos térmicos utilizados en la industria alimentaria se encuentra la fritura a vacío,la cual ha mostrado ser una alternativa para conseguir productos con características sensoriales deseadas, menor contenido de grasa y mayor pérdida de humedad. El objetivo de este estudio fue evaluar el contenido de grasa y humedad de chorizos con harina de garbanzo freídos a vacío. Para ello, se realizaron cuatro tratamiento: T1 (muestra control), T2, T3 y T4 con incorporación de harina al 3, 6, y $9 \%$, 
Autor a quien dirigir la correspondencias: Acevedo Correa*, D;. Correo electronico: diofanor3000@gmail.com respectivamente, de los cuales se tomaron los dos mejores sensorialmente (T1 y T4). El contenido de humedad de chorizos, se realizó en un horno a $105^{\circ} \mathrm{C}$ de acuerdo con el método de la (AOAC 1980).Para el contenido de grasa se utilizó el método de soxhlet. La fritura se realizó adiferentes temperaturas $(87.65,90,90,120,120,120,120,150,150$, $152.34^{\circ} \mathrm{C}$ ) con intervalos de tiempo de 4.0, 3.0, 5.0, 2.92, 4, 4, 5.07, 3, 5, 4 minutos. Se determinó que la mayor pérdida de humedad se dio a $152.34^{\circ} \mathrm{C} / 4.0$ minutos para $\mathrm{T} 4$, mientras que en $\mathrm{T} 1$ fue a $150{ }^{\circ} \mathrm{C} / 3$ minutos. Para la absorción de grasa, la mayor ocurrió a $87.65^{\circ} \mathrm{C}$ a 4 minutos para T1, mientras que T4 presentó un comportamiento diferente. El contenido de humedad y grasa para todas las corridas fueron inversamente proporcional al tiempo y temperatura de freído, por lo que se puede decir que esta técnica de freír reduce la absorción de aceite, además de conservar las características sensoriales del producto.

Palabras clave: Absorción de aceite, humedad y fritura a vacío.

\section{ABSTRACT}

Inside the thermal treatments used in the food industry one finds the frying to gap, which has showed to be an alternative to obtain products with sensory wished characteristics, contained minor of greasy and major loss of moisture. The target of this study was to evaluate the content of fat and moisture of sausages with flour of chick-pea fried to the gap. For it, four realized treatment: T1 (it shows control), T2, T3 and T4 with incorporation of flour to 3, 6, y $9 \%$, respectively, of which there took the best two sensory (T1 and T4). The content of moisture of sausages, it was realized in a microwave to $130^{\circ} \mathrm{C}$ in accordance with the method of (AOAC 1980). For the content of fat the method of soxhlet is 
used. The frying was realized to different temperatures $\left(87.65,90,90,120,120,120,120,150,150,152.34^{\circ} \mathrm{C}\right)$ with intervals of time of $4.0,3.0,5.0,2.92,4,4,5.07,3,5,4$ minutes. For the absorption of fat, the biggest happened to $87.65^{\circ} \mathrm{C}$ to 4 minutes for $\mathrm{T} 1$, whereas $\mathrm{T} 4$ presented a different behavior. The content of moisture and fat for all the bullfights they were inversely proportional to the time and temperature of fried, from what it is possible to say that this skill of frying reduces the absorption of oil, in addition to preserving the sensory characteristics of the product.

Key words: Absorption oil, frying on a vacuum, moisture

\section{INTRODUCCIÓN}

El freír los alimentos es uno de los procesos culinarios más antiguos, y fue seguramente uno de los primeros procesos técnicos que permitió prolongar la vida útil de los alimentos. Esta técnica culinaria corresponde a métodos de cocción de alimentos por medio de la inmersión en aceite comestible (principalmente de origen vegetal) o grasa caliente (mayoritariamente de origen vegetal o animal), por sobre el punto de ebullición del agua (Montes et al., 2016). El aceite actúa como transmisor de calor, produciendo un calentamiento rápido y uniforme del producto (Saguy y Dana, 2003), originando características sensoriales (color, textura y sabor) apreciadas por los consumidores (Villamizar et al., 2011).Sin embargo, la comprensión de la transferencia de grasa durante la fritura de productos cárnicos ha sido de interés para los procesadores ya que los consumidores se preocupan más por la cantidad de grasa que consumen, debido a su efecto negativo sobre la salud (Das et al., 2013). Cuando no se controlan las variables del proceso como la temperatura y tiempo se presentan efectos indeseables derivados de las altas temperaturas que participan en el proceso de fritura y la presencia de oxígeno en el proceso provoca la degradación de importantes compuestos nutricionales y la generación de moléculas tóxicas como la acrilamida(Villamizar et al., 2011; Seijas y Pabón, 2017).

Actualmente, en Colombia el consumo de alimentos fritos se ha convertido en una de 
las alternativas más viables para saciar el hambre en cualquier momento, sin embargo, los aportes nutricionales de estos alimentos no son $100 \%$ saludables ya que el contenido de grasa en su mayoría es relativamente alta dando como resultado que muchas personas se abstengan a consumirla (Gómez, 2011; Acevedo et al., 2017).La modificación de las formulaciones en la mayoría de los productos se ha hecho necesaria e importante en la industria alimentaria, con el fin de tener mayor rendimiento en cuanto costo, y darle un valor nutricional agregado a los productos. El uso de componentes orgánicos como los granos en derivados cárnicos se han utilizado como extensores, tal es el caso del garbanzo que permite el mejoramiento de la calidad proteica y el rendimiento del proceso como tal, además de disminuir el déficit que existe a escala nacional por la existencia de fuentes de proteína, el objetivo es sustituir parte la proteína animal por proteína vegetal, ofreciendoproteínas de alto valor biológico y funcionalidad adecuado (Albarracín et al., 2010); cabe resaltar que la incorporación de extensores cárnicos no siempre es para reemplazar parte de la proteína animal, sino también para sustituir gran parte de la grasa contenida en el producto (Torres et al., 2016).

La fritura por inmersión al vacío, ha mostrado ser una alternativa para conseguir productos con características sensoriales deseadas, mayor pérdida de agua y con menor contenido de grasa del que se obtiene en una fritura a presión atmosférica. Las posibles ventajas del proceso de fritura al vacío son la reducción de la absorción de grasa en el producto final, pérdida de humedad, preservación de los colores, sabores característicos del producto, disminución de la cantidad de acrilamida y conservación de los compuestos nutricionales; lo anterior es debido al control de la cantidad de oxígeno y bajas de temperaturas de operación (Montes et al., 2016). Por la tanto, el objetivo de este estudio fue evaluar el contenido de grasa y humedad de chorizos con incorporación de harina de garbanzo freídos al vacío.

\section{MATERIALES Y METODOS}

Los ingredientes utilizados fueron adquiridos de un supermercado local de la ciudad de Cartagena de Indias, Bolívar-
Colombia y trasladados a las plantas pilotos de Ingeniería de Alimentos de la Universidad de Cartagena-Piedra Bolívar. 


\section{Obtención de la harina de garbanzo}

Las semillas de garbanzo (Cicerarietinum) se seleccionaran teniendo en cuenta que estén sanas (enteras y sin picaduras de gorgojo); se secaron utilizando una incubadora a $60{ }^{\circ} \mathrm{C}$. Luego fueron molidas en un molino tradicional y pasadas por un tamiz de malla No. 40 hasta obtener una harina fina (Jaimes et al., 2014).

\section{Elaboración de chorizos}

La carne de res se pesó y se dividió en ocho porciones iguales. Fueron molidas en un picador de discos de $10 \mathrm{~mm}$ de espesor a una temperatura de $7{ }^{\circ} \mathrm{C}$ para evitar la desnaturalización de las proteínas. Del tocino se tomó el $15 \%$ del peso de la carne. Luego, se realizaron las formulaciones (ver tabla 1) para cada tratamiento ( $\mathrm{T} 1=$ muestra control, sin harina. T2 $=3 \%$ : sustitución de harina de garbanzo. $\mathrm{T} 3=6 \%$ : sustitución de harina de garbanzo. T4 = 9\%: sustitución de harina de garbanzo). La carne fue molida con todos los ingredientes, se embutió, se escaldo y posteriormente se sometió a un choque térmico para luego ser llevados a refrigeración por 16 horas para su maduración.

Tabla 1. Formulaciones de los chorizos control y con incorporación de harina de $C$. arietinum.

\begin{tabular}{|l|c|c|c|c|}
\hline $\begin{array}{l}\text { MATERIA } \\
\text { PRIMA }\end{array}$ & $\begin{array}{c}\text { T1 } \\
(\%)\end{array}$ & $\begin{array}{c}\text { T2 } \\
(\%)\end{array}$ & $\begin{array}{c}\text { T3 } \\
(\%)\end{array}$ & $\begin{array}{c}\text { T4 } \\
(\%)\end{array}$ \\
\hline CARNE DE RES & 85 & 85 & 85 & 85 \\
\hline $\begin{array}{l}\text { TOCINO DE } \\
\text { CERDO }\end{array}$ & 15 & 12 & 9 & 6 \\
\hline $\begin{array}{l}\text { HARINA DE C. } \\
\text { ARIETINUM }\end{array}$ & $\mathrm{N} / \mathrm{A}$ & 3,0 & 6,0 & 9,0 \\
\hline HIELO & 10 & 10 & 10 & 10 \\
\hline SAL & 1.6 & 1.6 & 1.6 & 1.6 \\
\hline $\begin{array}{l}\text { CONDIMENTO } \\
\text { DE CHORIZO }\end{array}$ & 1 & 1 & 1 & 1 \\
\hline PIMIENTA & 0,50 & 0,50 & 0,50 & 0,50 \\
\hline HUMO LIQUIDO & 0.15 & 0.15 & 0.15 & 0.15 \\
\hline $\begin{array}{l}\text { CEBOLLA } \\
\text { LARGA }\end{array}$ & 5 & 5 & 5 & 5 \\
\hline AJO & 1.5 & 1.5 & 1.5 & 1.5 \\
\hline NITRITO & 1.5 & 1.5 & 1.5 & 1.5 \\
\hline POLIFOSFATO & 0.4 & 0.4 & 0.4 & 0.4 \\
\hline $\begin{array}{l}\text { ÁCIDO } \\
\text { ASCÓRBICO }\end{array}$ & 0.045 & 0.045 & 0.045 & 0.045 \\
\hline
\end{tabular}

Fritura a vacío de chorizos con incorporación de harina de $C$. arietinum

Se realizó la fritura a vacío de las muestras de Chorizo con harina de C. arietinum en el equipo Gastrovac $\circledast$ (Cocina Internacional, Barcelona) con medidas: $40 \times 26 \times 46 \mathrm{~cm}$, capacidad máxima de 10.5 litros y tensión 220 V (García-Segovia et al., 2007). La presión de vacío máxima utilizada en el equipo fue de $30 \mathrm{kPa}$, a la cual la temperatura de ebullición del agua fue de $70^{\circ} \mathrm{C}$. Las temperaturas del proceso de fritura se observan en la tabla 2. Los tiempos de fritura fueron de 2.92 a 5.07 minutos (ver tabla 2), establecidos mediante ensayos preliminares. Los chorizos fueron escurridos en una canasta de malla metálica para su posterior análisis. 
Tabla 2. Tiempo y temperatura de los chorizos control y con incorporación de harina de $C$. arietinum freídos a vacío

\begin{tabular}{|c|c|c|}
\hline CORRIDAS & $\mathbf{T}\left({ }^{\circ} \mathbf{C}\right)$ & TIEMPO (MIN) \\
\hline $\mathbf{1}$ & 87,65 & 4 \\
\hline $\mathbf{2}$ & 90 & 3 \\
\hline $\mathbf{3}$ & 90 & 5 \\
\hline $\mathbf{4}$ & 120 & 2,92 \\
\hline $\mathbf{5}$ & 120 & 4 \\
\hline $\mathbf{6}$ & 120 & 4 \\
\hline $\mathbf{7}$ & 120 & 5,07 \\
\hline $\mathbf{8}$ & 150 & 3 \\
\hline $\mathbf{9}$ & 150 & 5 \\
\hline $\mathbf{1 0}$ & 152,34 & 4 \\
\hline
\end{tabular}

\section{Evaluación sensorial}

Para elegir a los dos mejores tratamientos se realizó una evaluación de la calidad sensorial de los chorizos con incorporación de $C$. arietinum procesados mediante fritura a vacío, para ello se utilizó una escala hedónica de cinco puntos, donde los panelistas indicarán su grado de aceptación en los parámetros de color, olor, sabor, crocancia, y grasosidad. Las categorías en la escala variarán desde "me gusta mucho" hasta "me disgusta mucho". Para la evaluación se empleó un panel no entrenado de 30 catadores.

\section{Determinación de humedad}

Se tomaron las dos mejores muestras del análisis sensorial y se determinó el contenido de humedad siguiendo el método de la (AOAC 1980). El método consiste en tomar cinco gramos de las muestras de chorizos y someterlas a desecación en un honor (CALLENGER eléctrico de $60 \mathrm{~cm}$ a $120 \mathrm{~V}$ ) a una temperatura de $105^{\circ} \mathrm{C}$ durante 24 horas o hasta alcanzar un peso constante. El contenido de humedad se calculó con la siguiente ecuación:

$$
\begin{aligned}
& \% \text { humedad }(h) \\
& =\frac{\text { peso muestra }- \text { peso muestra seca }}{\text { peso muestra }} \times 100
\end{aligned}
$$

\section{Determinación de grasa}

De igual forma se tomaron las dos mejores muestras del análisis sensorial y el contenido de grasa se determinó con $1 \pm$ $0,01 \mathrm{~g}$ de muestra utilizando un Extractor Soxhlet y Bencina de petróleo con punto de ebullición $60-80^{\circ} \mathrm{C}$, con reflujo de $8 \mathrm{~h}$, cada ensayo se realizó 3 veces; se recuperó el solvente a través de un evaporador rotario por destilación. EI extracto fue secado a $60^{\circ} \mathrm{C} \pm 0,1$ durante 30 $\min$ en un horno eléctrico (CALLENGER eléctrico de $60 \mathrm{~cm}$ a $120 \mathrm{~V}$ ) hasta eliminar el solvente, se enfrió y se pesó, los resultados se expresaron en base seca. El cálculo se realizó mediante la siguiente ecuación: 
$\%$ grasa

$=\frac{\text { peso vaso con grasa }- \text { peso del vaso vacio }}{\text { peso muestra }} \times 100$

\section{Análisis de datos}

El procesamiento de la información obtenida de los análisis se llevó a cabo a través del programa estadístico Statgraphics Centurión $\mathrm{XVI}$, en el cual se determinara la existencia 0 no de diferencias estadísticamente significativas de cada uno de los parámetros evaluados, a través de un análisis de varianza ANOVA completamente aleatorio y una prueba de Tukey con la que se detectaron diferencias entre los valores de las medias; diferencias significativas $p<0.05$.

\section{RESULTADOS Y DISCUSIÓN}

\section{Análisis sensorial}

En la tabla 3 se presentan los resultados del análisis sensorial de los chorizos con incorporación de harina de garbanzo fritos a vacío. El tratamiento T4 (con incorporación de harina de garbanzo al 9\%) presentó mayor grado de acepción en el color, olor y grasocidad, sin ninguna diferencia significativa en cuanto al color con respecto a T1. En aspectos como sabor y crocancia la mayor aceptación la obtuvieron T1 y T3, respectivamente. Por otra parte, se observa que a medida que aumenta el contenido de harina de garbanzo en los chorizos para las cuatro muestras el sabor fue disminuyendo su grado de aceptación, por tanto, se sugiere que a niveles altos de harina de garbanzo puede afectar la aceptación de los chorizos.

Tabla 3. Grado de aceptación del chorizo en los parámetros evaluados.

\begin{tabular}{|c|c|c|c|c|c|}
\hline $\begin{array}{c}\text { TRATA } \\
\text { MIENT } \\
0\end{array}$ & $\begin{array}{c}\text { COLO } \\
\mathbf{R}\end{array}$ & OLOR & $\begin{array}{c}\text { SABO } \\
\text { R }\end{array}$ & $\begin{array}{l}\text { CROC } \\
\text { ANCIA }\end{array}$ & $\begin{array}{c}\text { GRAS } \\
\text { OCIDA } \\
\text { D }\end{array}$ \\
\hline T1 & $\begin{array}{c}3,8 \pm \\
0,714 \\
b\end{array}$ & $\begin{array}{c}3,7 \pm \\
0,651 \\
b\end{array}$ & $\begin{array}{l}3,933 \pm \\
0,784^{b}\end{array}$ & $\begin{array}{c}3,38 \pm \\
0,961^{a} \\
b\end{array}$ & $\begin{array}{l}3,43 \pm \\
1,073^{b}\end{array}$ \\
\hline T2 & $\begin{array}{c}3,63 \\
\pm \\
0,76^{a}\end{array}$ & $\begin{array}{c}3,63 \\
\pm \\
0,80^{b}\end{array}$ & $\begin{array}{l}3,73 \pm \\
1,04^{\mathrm{ab}}\end{array}$ & $\begin{array}{c}3,26 \pm \\
0,86^{a}\end{array}$ & $\begin{array}{l}3,1 \pm \\
1,06^{a}\end{array}$ \\
\hline T3 & $\begin{array}{l}3,7 \pm \\
1,08^{a b}\end{array}$ & $\begin{array}{c}3,33 \\
\pm \\
1,02^{a}\end{array}$ & $\begin{array}{c}3,73 \pm \\
1,048^{a} \\
b\end{array}$ & $\begin{array}{l}3,2 \pm \\
1,12^{a}\end{array}$ & $\begin{array}{l}3,1 \pm \\
1,21^{a}\end{array}$ \\
\hline T4 & $\begin{array}{c}3,83 \\
\pm \\
0,87^{b}\end{array}$ & $\begin{array}{l}3,8 \pm \\
0,84^{b}\end{array}$ & $\begin{array}{c}3,66 \pm \\
1,12^{a}\end{array}$ & $\begin{array}{c}3,43 \pm \\
0,97^{b}\end{array}$ & $\begin{array}{c}3,76 \pm \\
0,97^{c}\end{array}$ \\
\hline
\end{tabular}

Letras diferentes en una misma columna indican diferencias estadísticamente significativas $(p<0,05)$.

Pérdida de humedad y absorción de grasa de los chorizos

El cálculo de la pérdida de humedad se realizó de acuerdo a la metodología anteriormente descrita y está determinado por los parámetros de temperatura y tiempo. En la tabla 4 se muestran los resultados para los dos mejores tratamientos de la evaluación sensorial (T1 y T4), se observa que independientemente de la muestra, el 
contenido de humedad en los chorizos se ve afectada por la intensidad del proceso, a medida que se manejan los valores máximos de temperaturas la pérdida de humedad se va haciendo significativamente mayor $(p<0,05)$ Sin embargo, las muestras de T4 presentaron menor pérdida con respecto a $T 1$, este hecho se presentó debido a la capacidad de ligamento de agua de las proteínas de la harina de garbanzo (Aguilar-Raymundo y Velez-Ruiz, 2013).

Tabla 4. Pérdida de humedad y absorción de grasa de los chorizos con mayor grado de aceptación sensorial.

\begin{tabular}{|c|c|c|c|c|c|}
\hline \multirow[b]{2}{*}{$\begin{array}{c}\mathrm{T} \\
\left({ }^{\circ} \mathrm{C}\right)\end{array}$} & \multirow[b]{2}{*}{$\begin{array}{l}\text { TIME } \\
(\min )\end{array}$} & \multicolumn{2}{|c|}{$\begin{array}{c}\text { PÉRDIDA } \\
\text { HUMEDAD (\%) }\end{array}$} & \multicolumn{2}{|c|}{$\begin{array}{l}\text { ABSORCIÓN } \\
\text { GRASA (\%) }\end{array}$} \\
\hline & & $\begin{array}{c}\text { T1 } \\
\text { (CONTROL) }\end{array}$ & T4 & $\begin{array}{c}\text { T1 } \\
\text { (CONTROL) }\end{array}$ & T4 \\
\hline $\begin{array}{c}87,6 \\
5 \\
\end{array}$ & 4 & $\begin{array}{c}43,3 \pm \\
1,0^{\mathrm{a}} \\
\end{array}$ & $\begin{array}{c}41,9 \pm \\
0,3^{\mathrm{a}} \\
\end{array}$ & $\begin{array}{c}43,5 \pm \\
1,0^{\mathrm{a}} \\
\end{array}$ & $\begin{array}{l}36 \pm \\
0,2^{a}\end{array}$ \\
\hline 90 & 3 & $\begin{array}{c}45,7 \pm \\
0,3^{\mathrm{b}} \\
\end{array}$ & $\begin{array}{c}44,5 \pm \\
1,3^{\mathrm{a}} \\
\end{array}$ & $35 \pm 0,4^{b}$ & $\begin{array}{c}34,5 \pm \\
0,5 b^{b}\end{array}$ \\
\hline 90 & 5 & $\begin{array}{c}54,9 \pm \\
0,1^{\mathrm{c}} \\
\end{array}$ & $\begin{array}{l}49 \pm \\
1,2^{b} \\
\end{array}$ & $\begin{array}{c}33,5 \pm \\
0,22^{b} \\
\end{array}$ & $\begin{array}{l}32 \pm \\
0,3^{c}\end{array}$ \\
\hline 120 & 2,92 & $\begin{array}{c}55,2 \pm \\
0,2^{\text {cd }} \\
\end{array}$ & $\begin{array}{c}51,3 \pm \\
0,3^{\text {bc }}\end{array}$ & $32 \pm 0,9^{b}$ & $\begin{array}{r}31,5 \\
\pm 0,5^{d} \\
\end{array}$ \\
\hline 120 & 4 & $\begin{array}{c}57,3 \pm \\
0,9^{\mathrm{e}} \\
\end{array}$ & $\begin{array}{c}53,1 \pm \\
0,3^{c} \\
\end{array}$ & $30 \pm 1,0^{c}$ & $\begin{array}{r}29,5 \\
\pm 0.8^{\text {de }} \\
\end{array}$ \\
\hline 120 & 4 & $\begin{array}{l}57,1 \pm \\
0,9 \mathrm{de}\end{array}$ & $\begin{array}{c}52,8 \pm \\
0,6^{c} \\
\end{array}$ & $29 \pm 1,0^{\mathrm{cd}}$ & $\begin{array}{l}28 \pm \\
0,6^{e}\end{array}$ \\
\hline 120 & 5,07 & $60,1 \pm 0,1^{f}$ & $\begin{array}{c}59,8 \pm \\
1,2^{\text {de }}\end{array}$ & $\begin{array}{c}25 \pm \\
1,01^{\mathrm{de}}\end{array}$ & $\begin{array}{c}24 \pm \\
0,2^{f} \\
\end{array}$ \\
\hline 150 & 3 & $61 \pm 0,6^{f g}$ & $\begin{array}{l}57,54 \\
\pm 0,3^{d} \\
\end{array}$ & $\begin{array}{c}24,5 \pm \\
0,5^{\mathrm{ef}}\end{array}$ & $\begin{array}{c}22,5 \pm \\
0,8^{f}\end{array}$ \\
\hline 150 & 5 & $\begin{array}{c}65,3 \pm \\
0,3^{\mathrm{h}} \\
\end{array}$ & $\begin{array}{c}59,4 \pm \\
1,2^{\text {de }} \\
\end{array}$ & $23 \pm 0,3^{f}$ & $\begin{array}{c}20,5 \pm \\
0,5^{\mathrm{g}} \\
\end{array}$ \\
\hline $\begin{array}{c}152 \\
34\end{array}$ & 4 & $\begin{array}{c}62,5 \pm \\
1,19 \\
\end{array}$ & $\begin{array}{l}61 \pm \\
1,3^{\mathrm{e}} \\
\end{array}$ & $22 \pm 1^{g}$ & $\begin{array}{c}18,5 \pm \\
0,5^{\mathrm{g}}\end{array}$ \\
\hline
\end{tabular}

Letras diferentes en una misma columna indican diferencias estadísticamente significativas $(p<0,05)$.

Por otra parte se observa que en el mismo tiempo de fritura (4 minutos) se presentaron diferencias estadísticamente significativas $(p<0,05)$ con respecto a la temperatura; a pesar de la variación de los parámetros de proceso, el factor que más influyó en la deshidratación de los chorizos después de la fritura al vacío fue la temperatura, si bien es claro que estos dos parámetros influyen directamente con la pérdida de humedad (Moreira, 2001). Aunque se cuenta con poca información bibliográfica del efecto de fritura a bajas presiones en productos cárnicos se han reportado investigaciones del efecto de este tipo de deshidratación en frutas y tubérculos; Crosa et al., (2014) evaluaron atributos fisicoquímicos de chips de papa elaborados por fritura al vacío y fritura tradicional, los valores en la pérdida de humedad no presentaron diferencias significativas, en comparación con el contenido de grasa donde las bajas presiones lograron reducir en promedio un $50 \%$ de materia grasa. Resultados similares obtuvieron Urbano et al., (2012) y Villamizar et al., (2011) los cuales estudiaron el efecto de ambas frituras (al vacío y tradicional)en chips de yuca y pasa-bocas de mango, respetivamente.

El efecto de la temperatura y tiempo en la absorción de grasa de los chorizos fritos al vacío presentaron diferencias estadísticamente significativa cuando se pasa de $87,65{ }^{\circ} \mathrm{C}$ a $152,34{ }^{\circ} \mathrm{C}$. El chorizo con incorporación de harina de garbanzo al 9 \% (T4) presento menor absorción de 
grasa con respecto a $T 1$, esto se debe principalmente a que los poros de la matriz alimentaria no están disponibles en su totalidad por la asociación existente entre proteína-carbohidratos. El efecto del vacío provocado por las bajas presiones hace que la temperatura necesaria para que el agua contenida en el alimento llegue al punto de ebullición sea menor, lo anterior respetando la ley de la termodinámica que establece una relación directamente proporcional entre el tiempo y la temperatura, este hecho ocasiona que la matriz alimentaria llegue al fin del proceso en menor tiempo en comparación con la fritura atmosférica, lo que explica una menor exposición del

\section{CONCLUSIÓN}

La fritura a vacío ha despertado el interés en investigadores ya que promete brindar productos sanos y seguros, por ello, se hace necesario utilizarla en diferentes matrices alimentarias. Los chorizos fritos a vacío con incorporación de harina de garbanzo pueden traer ventajas al producto alimento en el aceite y por lo tanto una disminución en la absorción de aceite. Lo anterior concuerda con los resultados obtenidos por Díaz et al., (2016).

Otros parámetros a considerar en la absorción de grasa son la forma del alimento, la relación entre el tamaño inicial del producto y la superficie expuesta al medio circundante, las propiedades del aceite, las reacciones químicas, el contenido de humedad de los alimentos, la fase de enfriamiento, entre otros. (Teruel et al., 2014). Así, Urbano et al., (2012) exponen que al incrementar la temperatura la velocidad de deshidratación aumenta, reduciéndose el tiempo de fritura. ya que conserva el color y sabor característico, sin embargo, un aumento en el contenido de harina de garbanzo se puede afectar el sabor de estos. La fritura a $152{ }^{\circ} \mathrm{C}$ a vacío mostro una mayor pérdida de humedad y una menor absorción de aceite.

\section{REFERENCIAS BIBLIOGRAFICAS}

Acevedo Correa D., Montero Castillo P., Beltrán Cotta L., Gallo García L., Rodríguez Meza J. (2017). Efecto de la fritura al vacío sobre la absorción de aceite en empanadas de maíz (zea mays). Revista @limentech, Ciencia y Tecnología Alimentaría. ISSN: 1692-7125. Volumen $15 \mathrm{~N}^{\circ} 1$. Pp. 42 - 49. 
Aguilar-Raymundo, V. G., \& Velez-Ruiz, J. F. (2013). Propiedades nutricionales y funcionales del garbanzo (Cicer arietinum L.). Temas Selectos de Ingeniería de Alimentos, 7, 25-34.

Albarracín, W., Acosta, L. F., \& Sánchez, I. C. (2010). Elaboración de un producto cárnico escaldado utilizando como extensor harina de fríjol común (Phaseolus spp.). Vitae, 17(45), 264271.

Crosa, M. J., Elichalt, M., Skerl, V., Cadenazzi, M., Olazabal, L., \& Roberto, S. (2014). Chips de papa, la fritura en vacío y beneficios para la salud. Revista de Laboratorio Tecnológica de Uruguay, 9, 70-74. Retrieved from http://nutricion.edu.uy/u01/uploads/201 6/06/6.-Chips-de-papa-fritura-en-vacioy-salud-2014.pdf

Das, R., Pawar, D. P., \& Modi, V. K. (2013). Quality characteristics of battered and fried chicken: Comparison of pressure frying and conventional frying. Journal of Food Science and Technology, 50(2), 284-292.

https://doi.org/10.1007/s13197-0110350-z

Díaz, W., Romero, P., Castelanos, F., Rodriguez, J., Gutierrez, L., \& Restrepo, S. (2016). Efecto de la presión a vacío en la fritura por inmersión de hamburguesas.

Agronomía

Colombiana, 34(1), 371-373. https://doi.org/10.15446/agron.colomb. v34n1 supl.58134

Gómez, C. (2011). La industria gastronómica en Colombia sigue creciendo. Retrieved from http://www.portafolio.co/economia/finan zas/industria-gastronomica-colombiasigue-creciendo-141936

Jaimes, J., Restrepo, D., \& Acevedo, Di. (2014). Preparation and determination of the functional properties of the trupillo proteic concentrated (prosopis juliflora). biotecnología en el sector agropecuario y agroindustrial, 12(1), 144-152.

Montes, O. N., Millar, M. I., Provoste, L. R., Martínez, M. N., Fernández, Z. D., \& Morales, g. (2016). Absorción de aceite en alimentos fritos. rev chil nutr, 43(1), 87-91. https://doi.org/10.4067/s071775182016000100013.

Moreira, R. G. (2001). Deep-fat frying of foods. in j. m. irudayaraj (ed.), food processing operations modeling: design and analysis (pp. 115-117). new york: marcel dekker, inc.

Saguy, I. S., \& Dana, D. (2003). integrated approach to deep fat frying: 
engineering, nutrition, health and consumer aspects. journal of food engineering, 56(2-3), 143-152. https://doi.org/10.1016/s0260-

8774(02)00243-1

Seijas Ch., Héctor.y Pabón M., Carolina. (2017). Coeficiente de difusión y pérdida de humedad durante el proceso de pre-fritura de la arracacha (Arracacia xanthorrhiza). Revista @limentech, Ciencia y Tecnología Alimentaría. ISSN:1692-7125. Volumen $15 \mathrm{~N}^{\circ} 1$. Pp. $17-27$.

Teruel, M. R., García-Segovia, P., MarínezMonzó, J., Linares, M. B., \& Garrido, M. D. (2014). use of vacuum-frying in chicken nugget processing. innovative food science and emerging technologies, 26, 482-489. https://doi.org/10.1016/j.ifset.2014.06.0 05

Torres, J., González, K., Acevedo, D., \& Jaimes, J. del C. (2016). Efecto de la utilización de harina de Lens culinaris como extensor en las características físicas y aceptabilidad de una salchicha. Revista Tecnura, 20 (49), 15. https://doi.org/10.14483/udistrital.jour.te cnura.2016.3.a01

Urbano, A. M., García, P., \& Martínez, J. (2012). Evaluacion del comportamiento de yuca (Manhiot CHIPS, 1-20).

Villamizar, R. H., Quiceno, M. C., \& Giraldo, G. A. (2011). en la obtención de pasabocas de mango (manguifera indica l.) comparison of atmospheric and vacuum frying in obtaining snack of mango (Manguifera indica L.). 